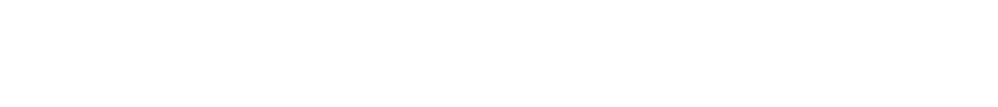 ÖÄ̈ $p^{\circledR}$ Õ̂ि ÁÁ $\quad$ ¿ Áá

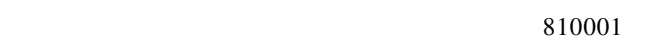

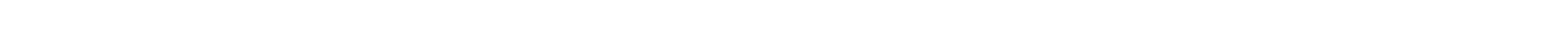

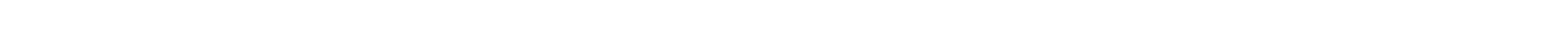

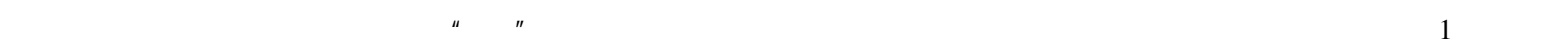

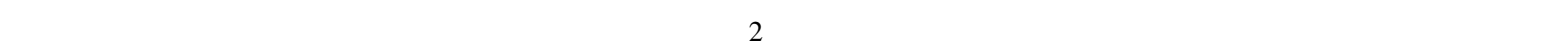
๒Đî a

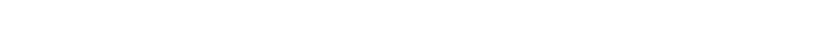

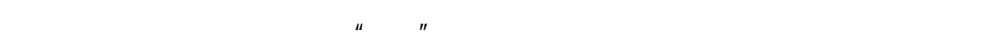

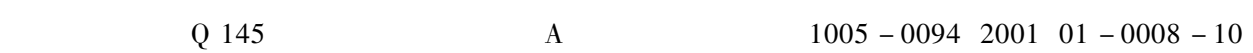

\section{Evolutionary mechanisms of species coexistence in ecological communities}

ZHANG Xiao-Aif-ZHAO Liang $\mathrm{f}-\mathrm{KANG}$ Ling

Northwest Plateau Institute of Biology $\mathbf{f - C h i n e s e ~ A c a d e m y ~ o f ~ S c i e n c e s f - X i n i n g ~} 810001$

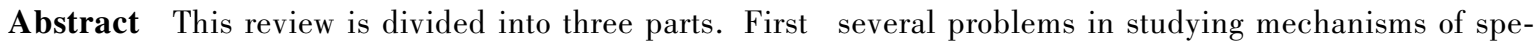
cies coexistence in ecological communities are generalized. Secondf-zome concepts explaining plant and animal species coexistence are reviewed. Within plant communitiesf these concepts include environmental factorsf $f$ seed re-immigration $f$ - miche differentiation $f$ toalanced competition $f$-species-poolf-regeneration nichef-oexistence of ecologically similar species and the null community. In animal communitiesf the effects of patchfedgef $\mathbf{f o n g r e g a t i o n} f$-dispersionf ty are discussed. Finallyf
\end{abstract}

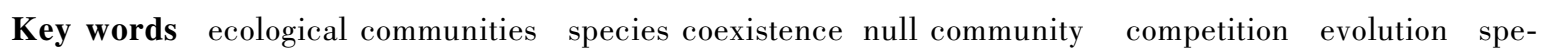
cialization $f$ optimal foraging theory

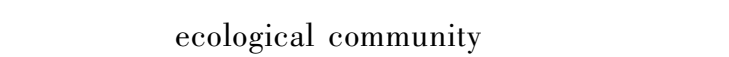

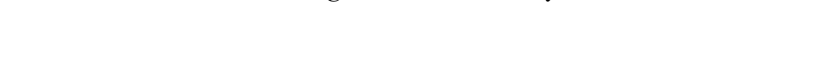

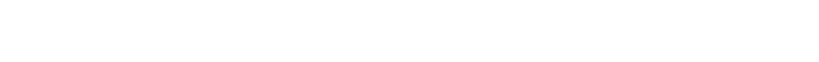

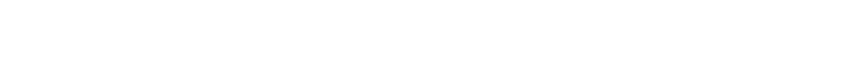

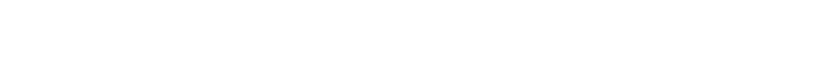

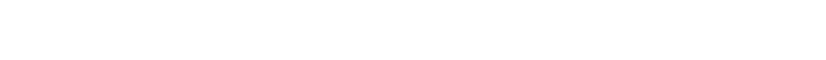

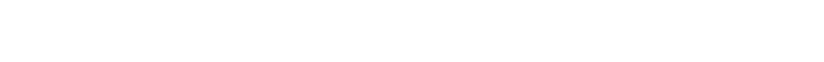

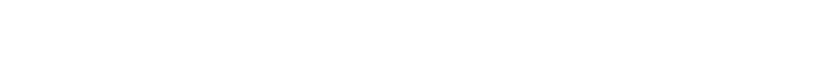

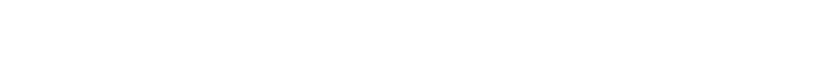

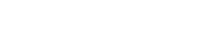

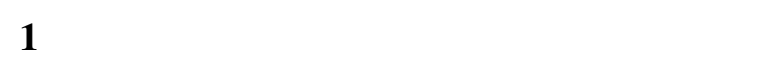

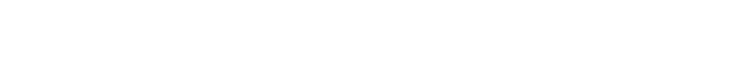

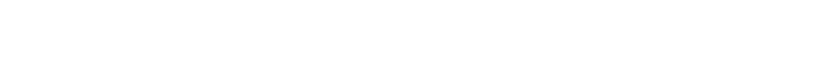

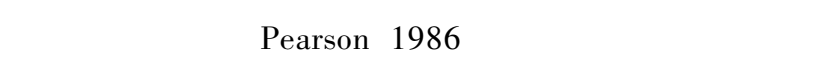

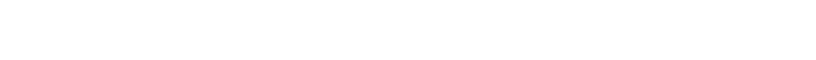

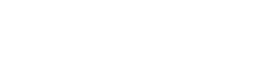

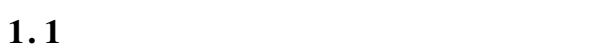

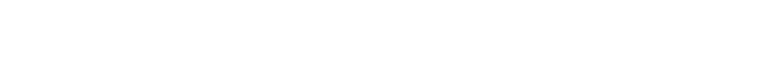

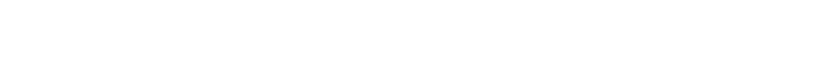

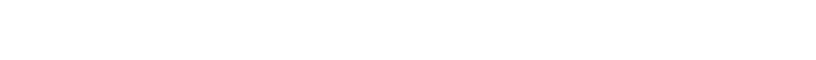

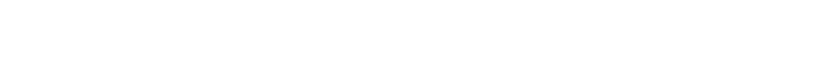

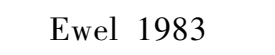

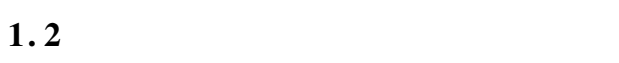

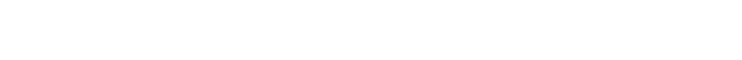

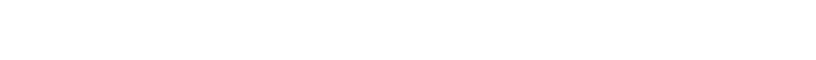




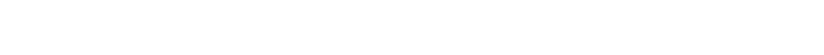

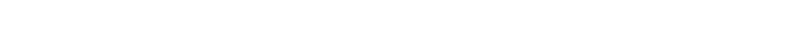

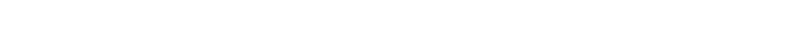

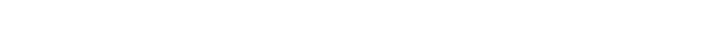

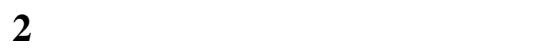

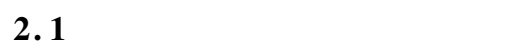

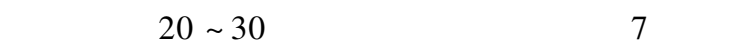

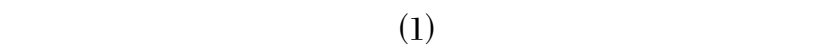

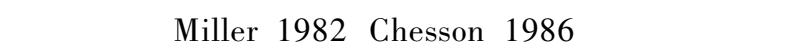

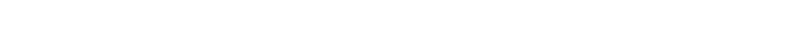

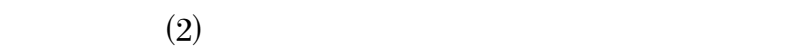

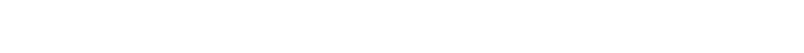

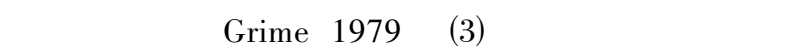

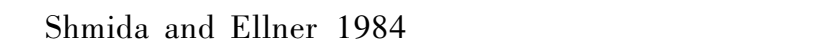

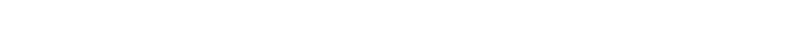

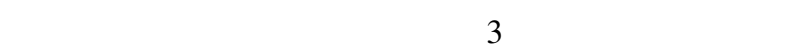

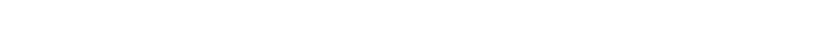

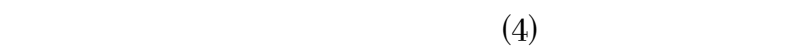

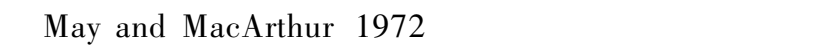

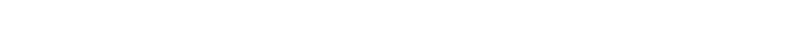

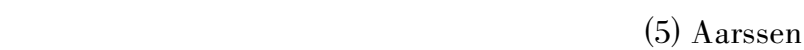

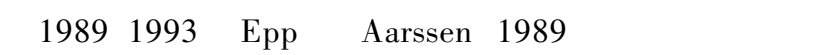

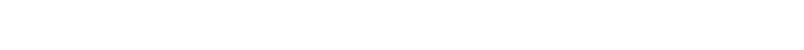

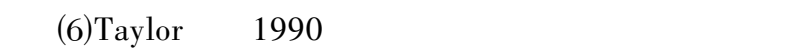

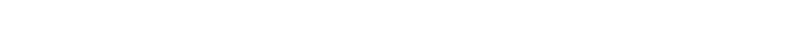

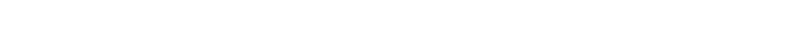

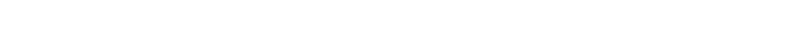

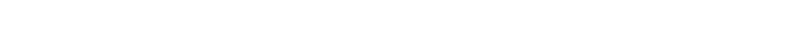

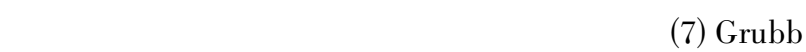

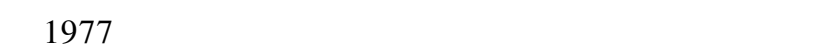

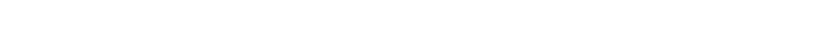

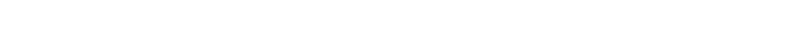

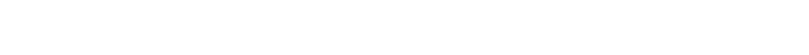

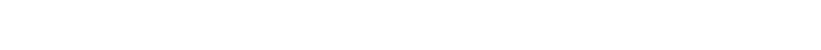

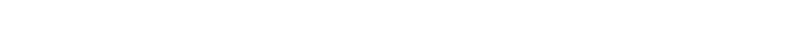

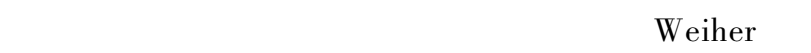

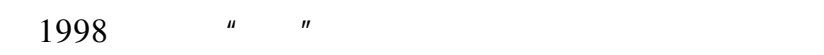

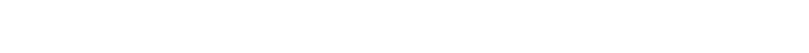

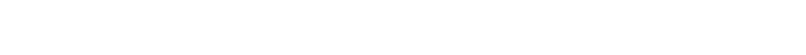

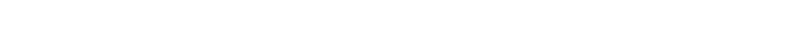

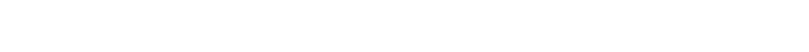

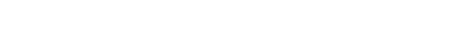

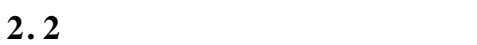

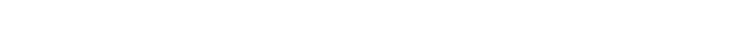

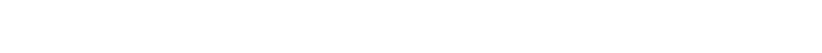

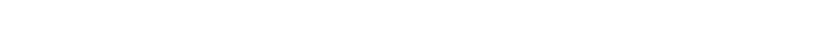

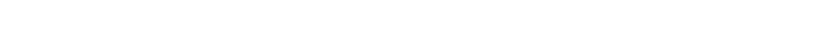

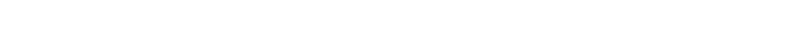

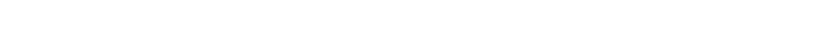

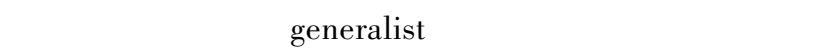

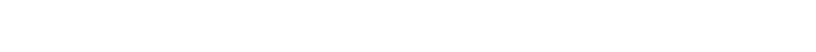

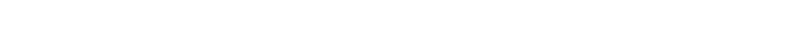

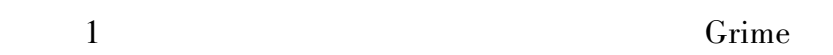

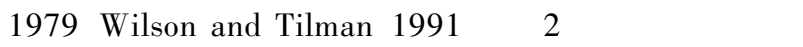
ÑßĐ

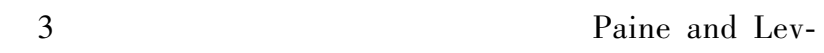

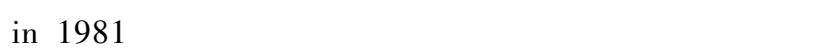

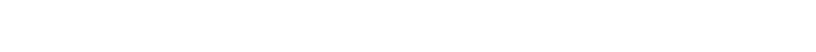

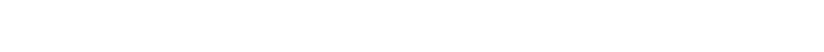

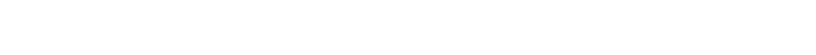

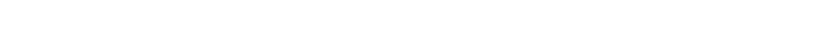

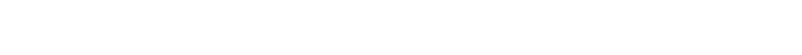
İ Â£Æ£Á Ò

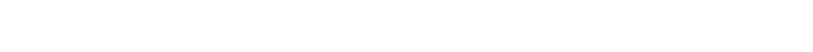

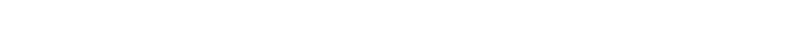

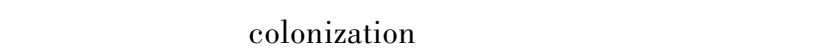

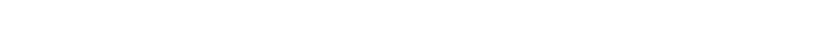

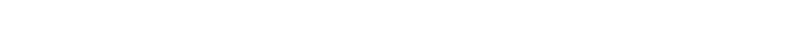

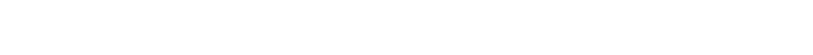

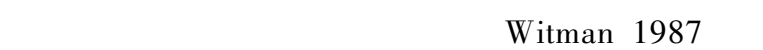

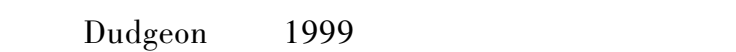

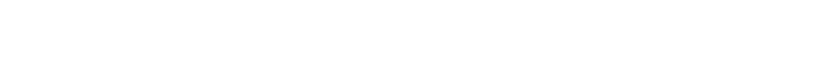

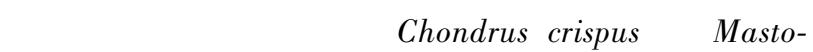

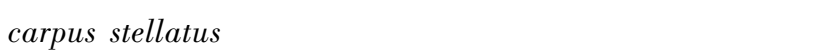

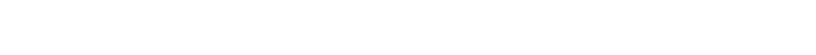

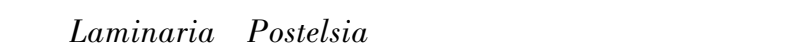

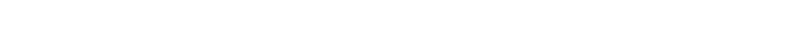

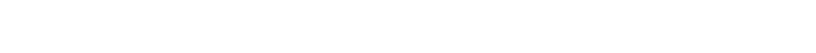

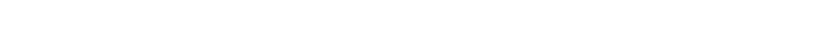

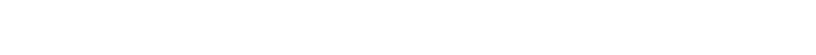

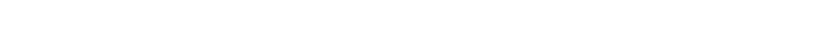

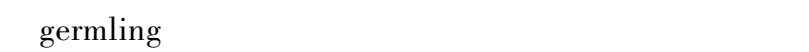

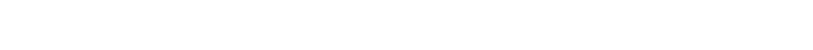




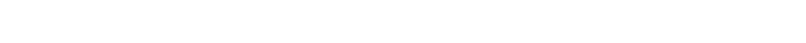

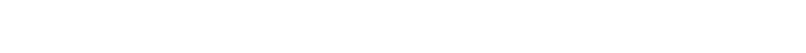

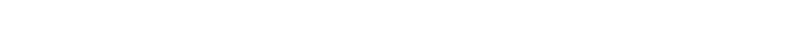
Ơ ĆÉ ó

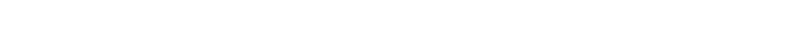

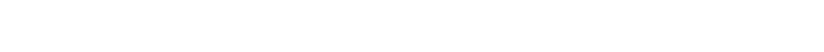

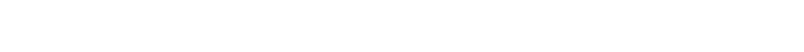

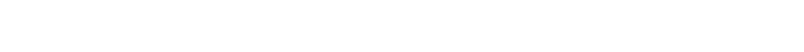

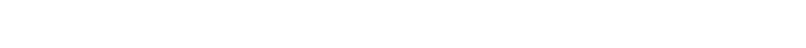

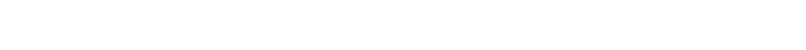

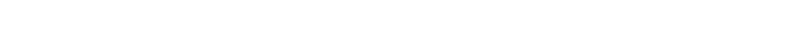

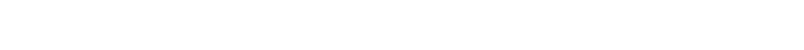

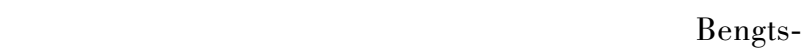

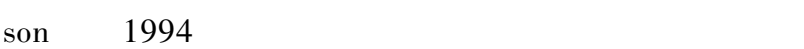

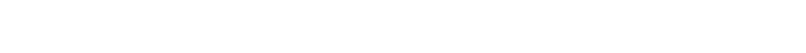

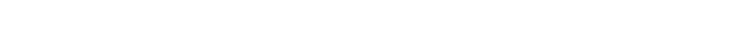

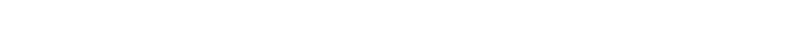

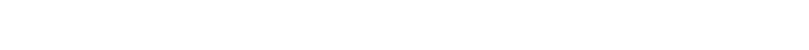

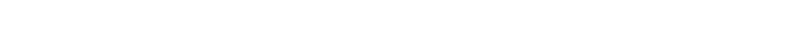

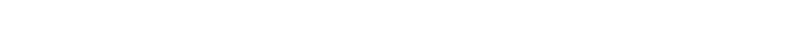

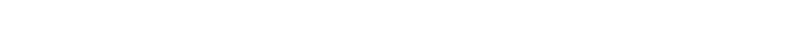

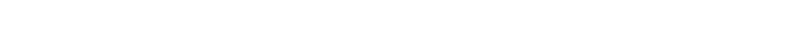

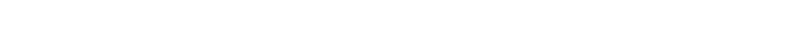

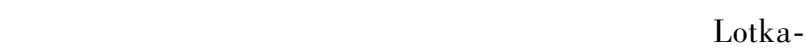

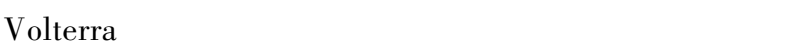

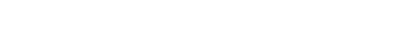

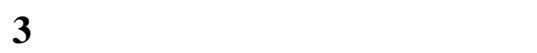

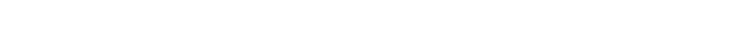

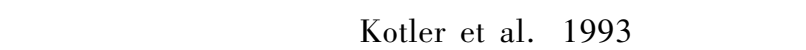

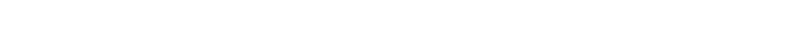

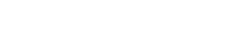

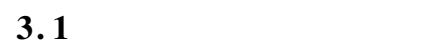

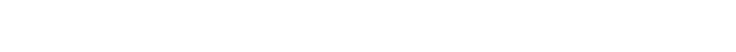

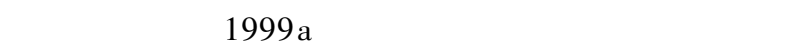

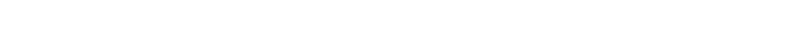

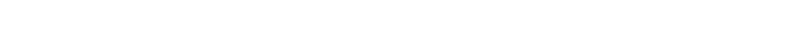

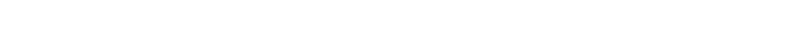

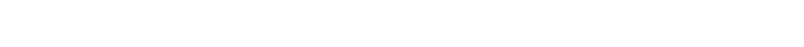

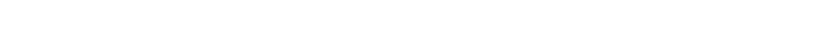
ỐAล̈Ëû

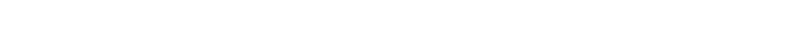

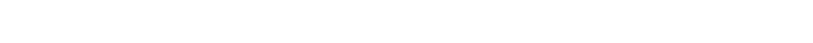

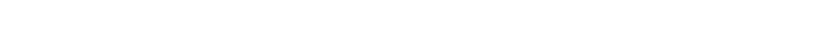

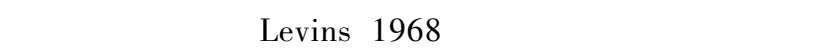

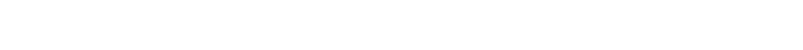

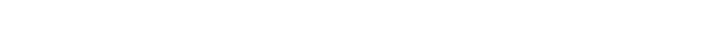

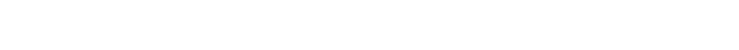

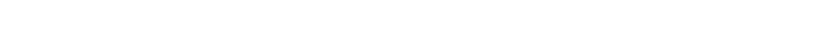

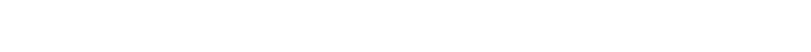

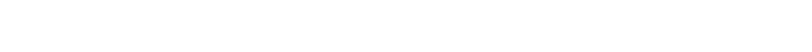

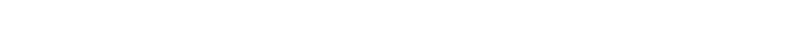

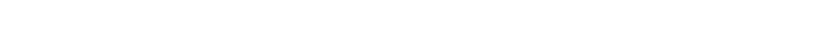

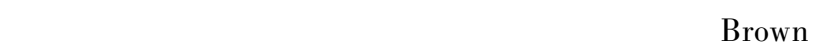

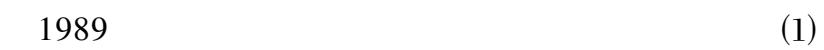

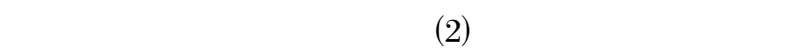
» ỐxÊÔ' · á, »

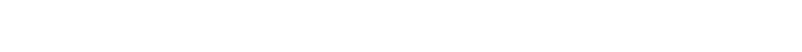

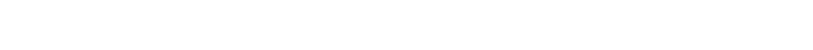

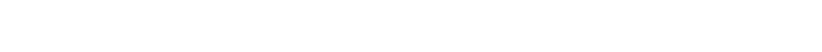

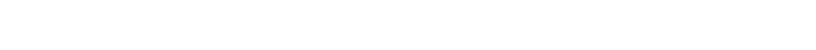

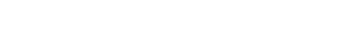

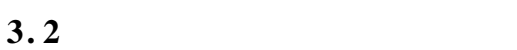

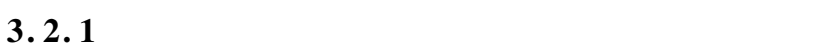

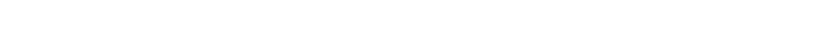

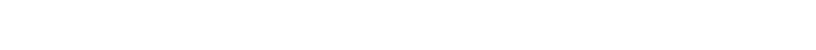

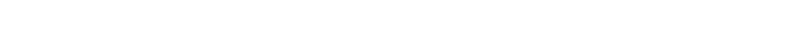

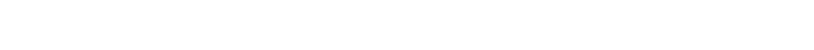

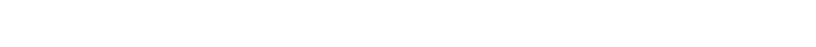

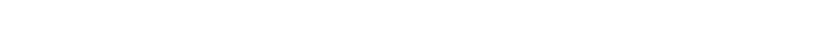

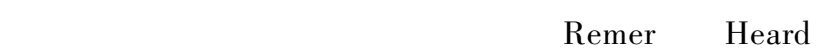

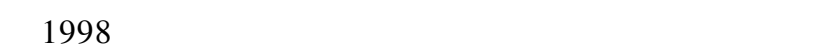

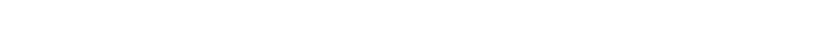

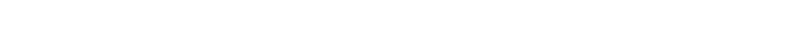

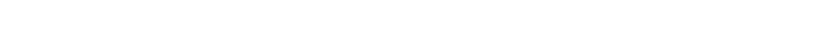

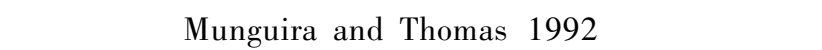

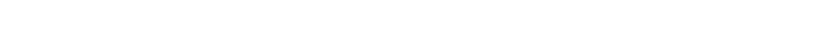

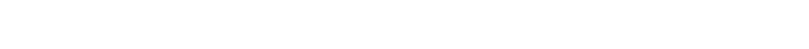

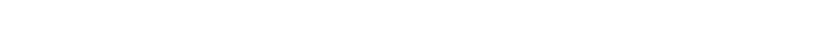

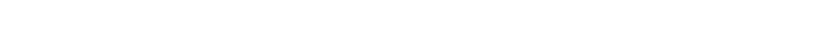

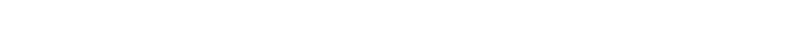

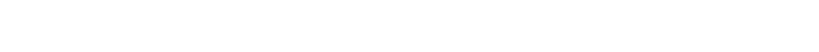

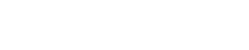




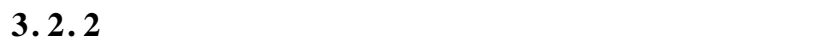

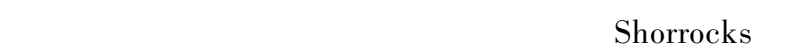

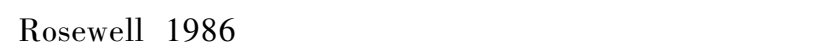

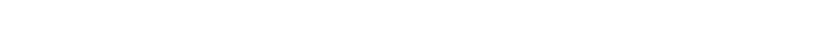

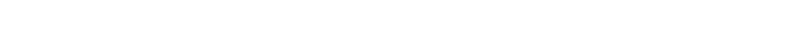

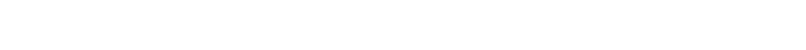

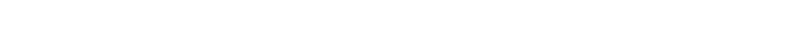

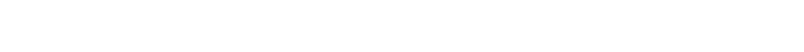

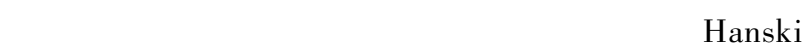

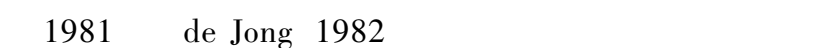

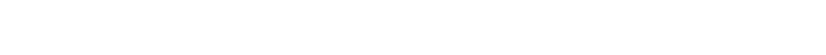

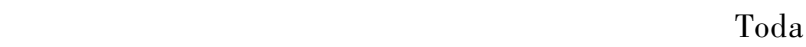

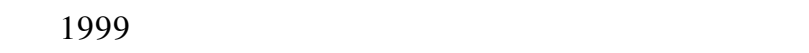

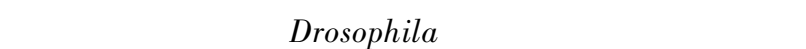

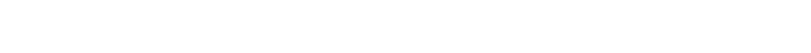
\# Ёù

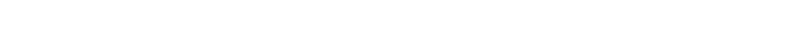
İ à

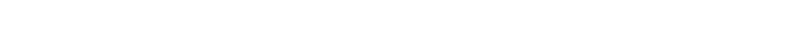

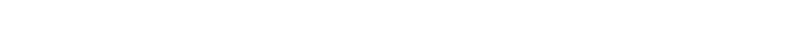

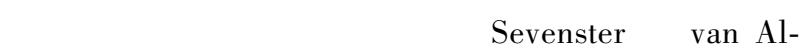

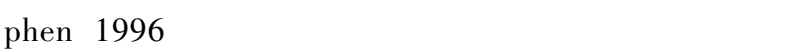

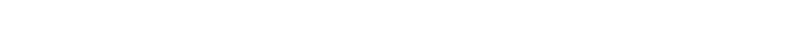

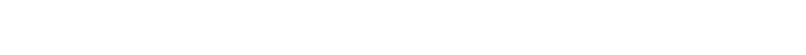

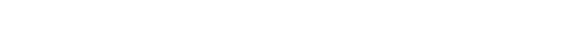

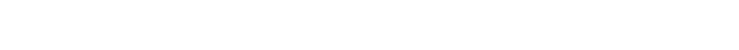

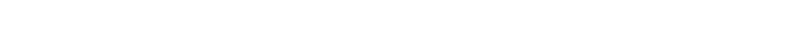

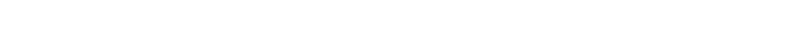

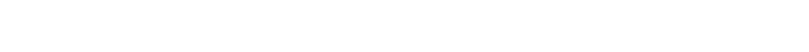

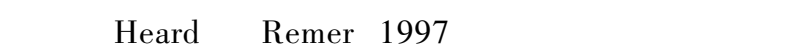

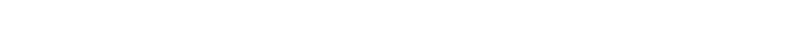

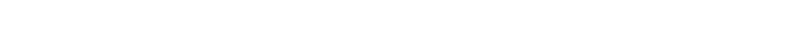

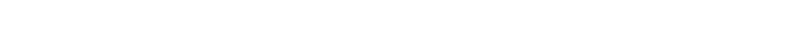

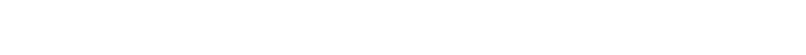

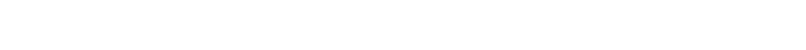

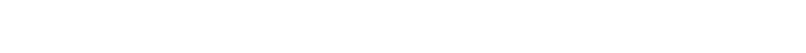

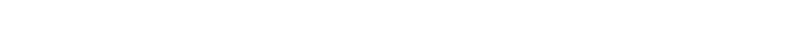

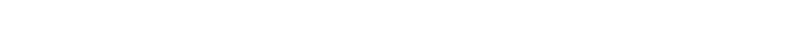

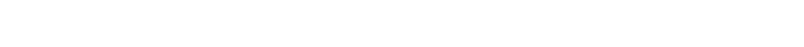

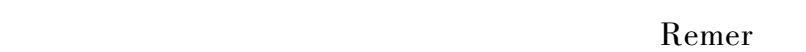

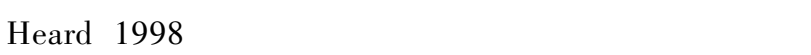

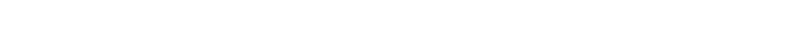

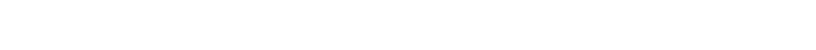

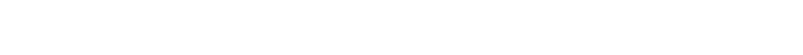

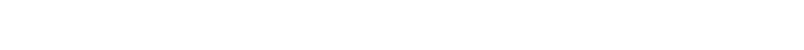

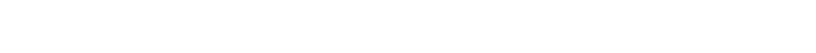

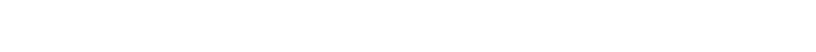

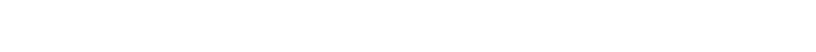

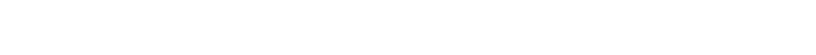

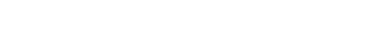

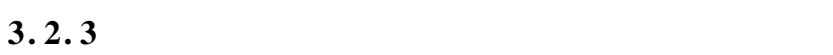

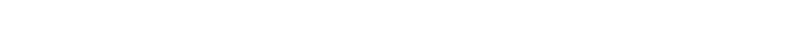

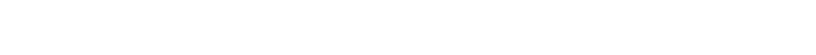

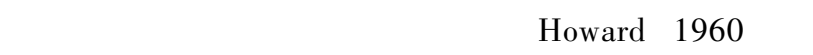

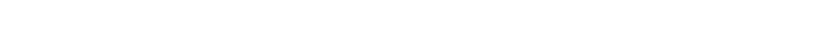

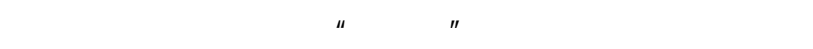

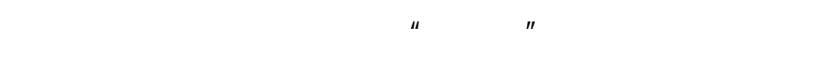

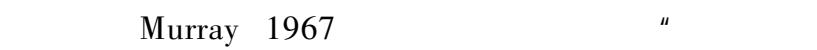

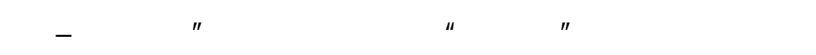

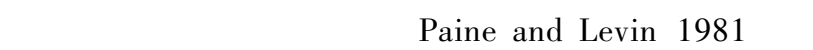

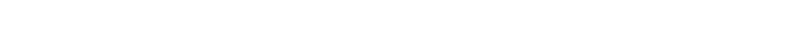

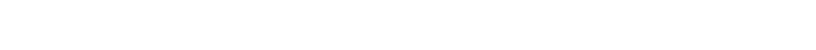

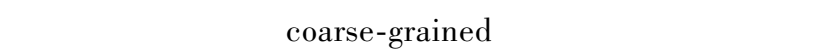

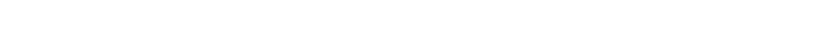

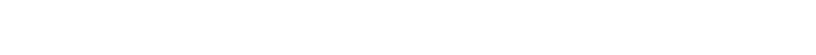

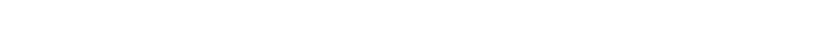

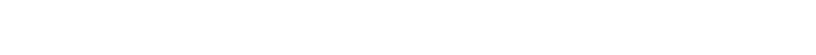

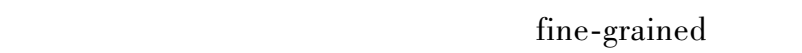

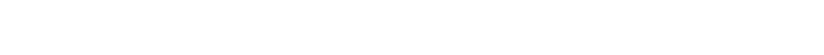

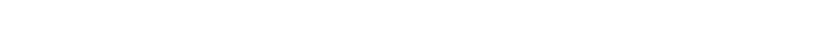

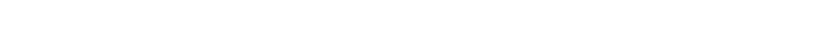

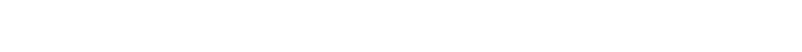

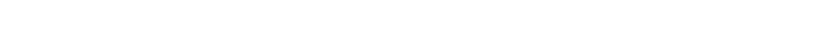

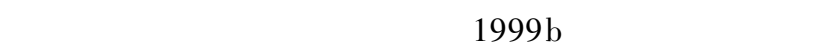

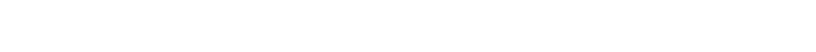

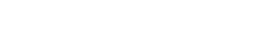

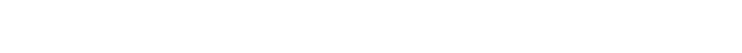

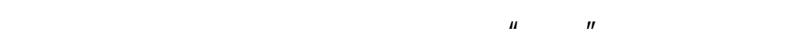

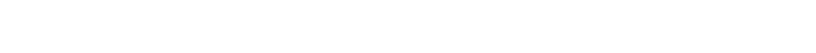

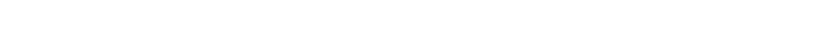

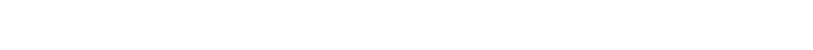

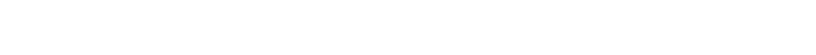

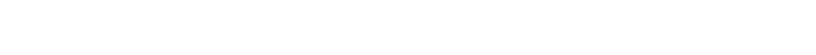

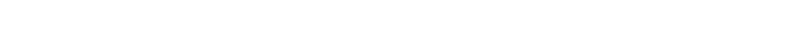




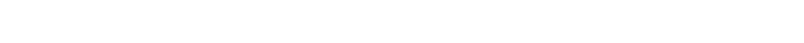

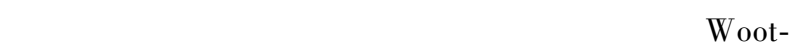

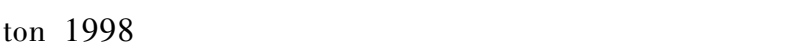
$\mu$

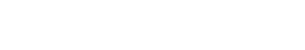

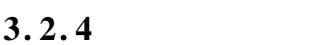

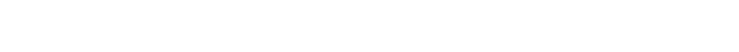

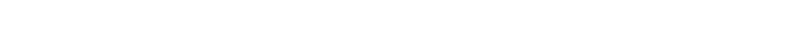

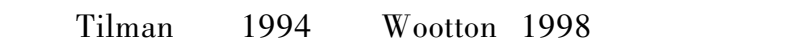

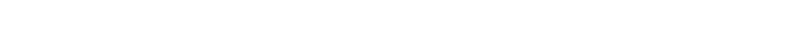

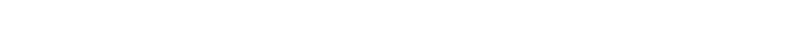

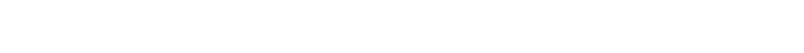

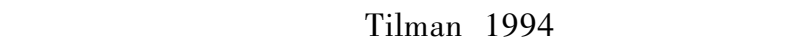

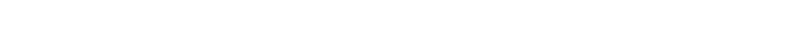
"

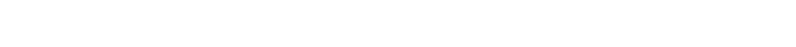

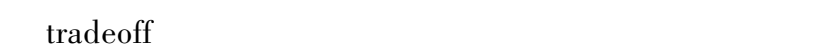

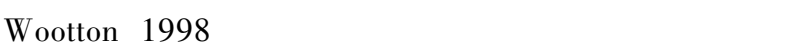

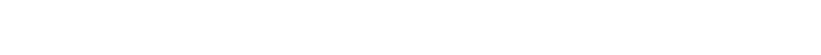

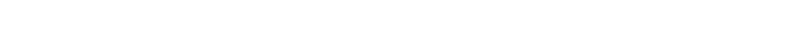

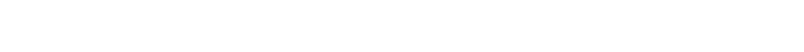

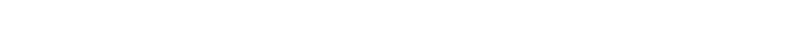

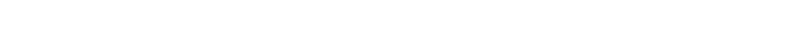

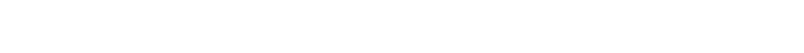

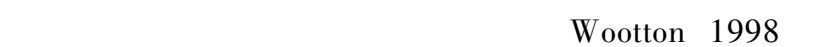

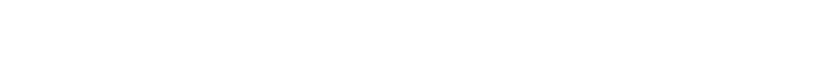

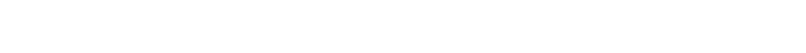

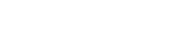

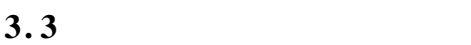

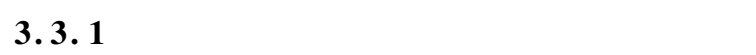

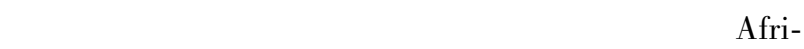

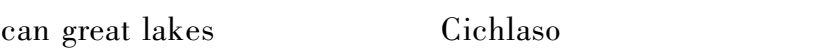

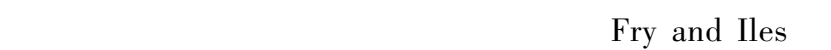

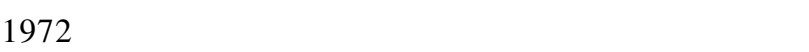

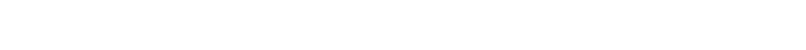
»"

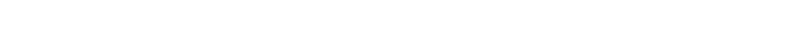

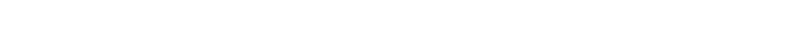

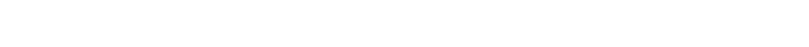

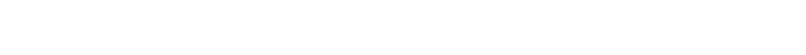

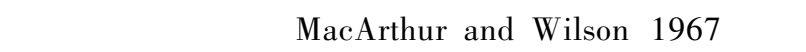

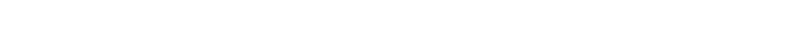

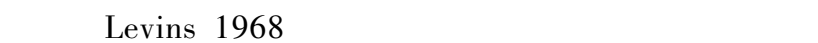

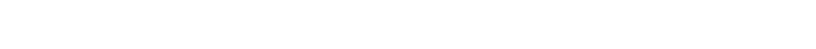

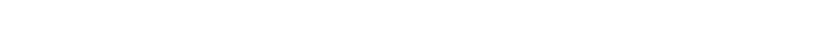

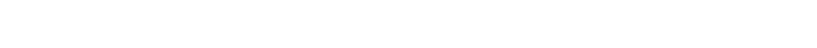

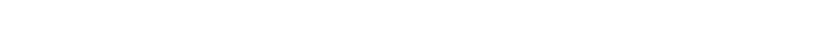

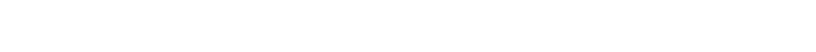

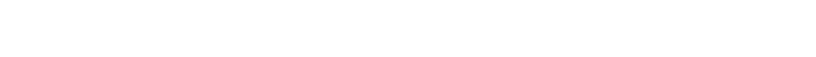

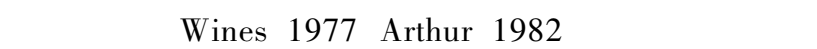

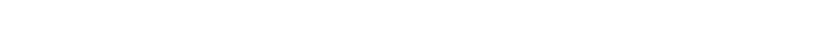

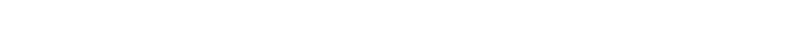

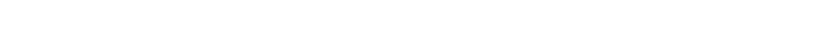

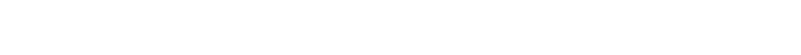

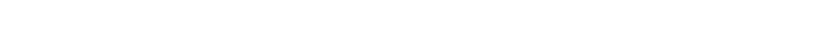

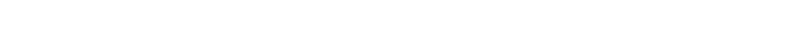

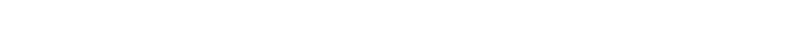

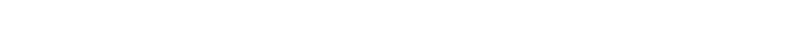

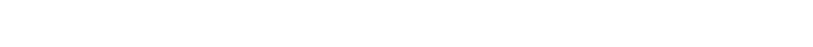

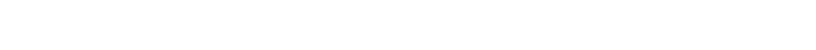

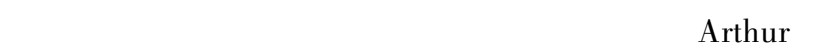
$1982 \mathrm{f} \Subset E$

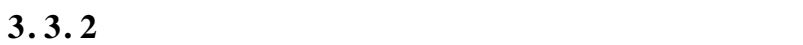

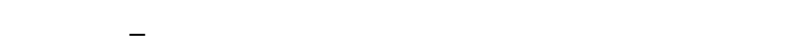

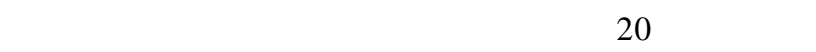

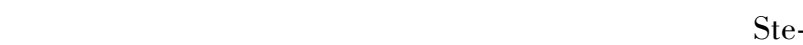

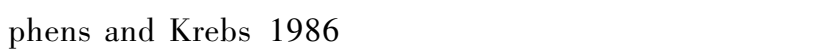

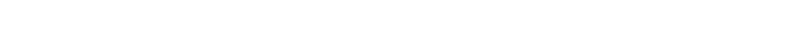

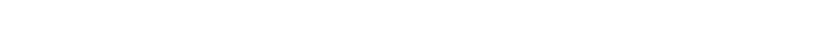

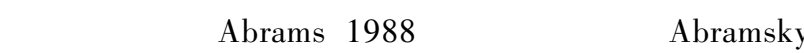

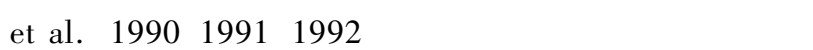

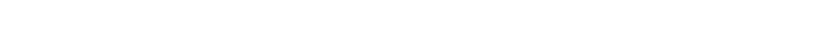

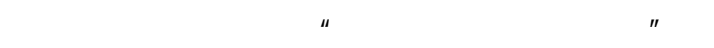

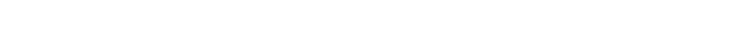

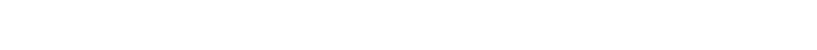
Ẻ À̀ ÂÛ̈ Î

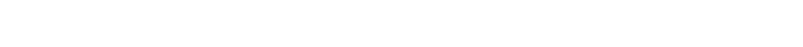

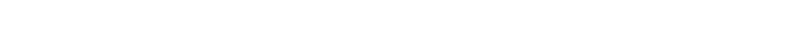

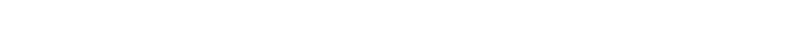

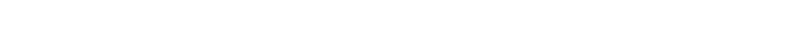

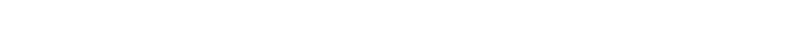

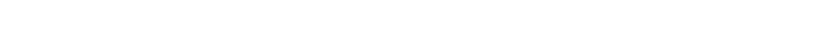

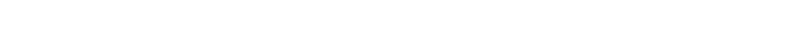

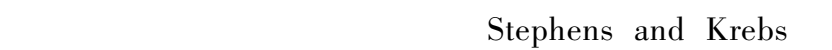




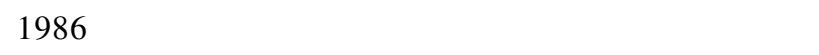

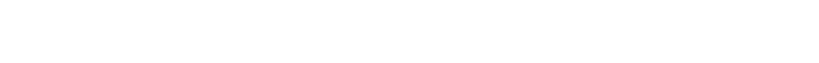

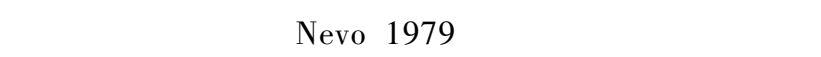

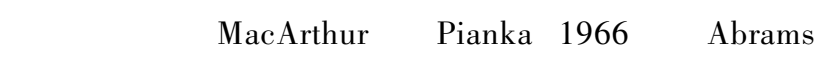

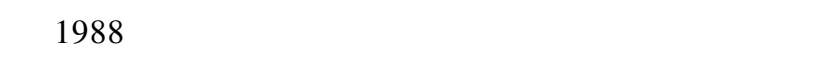

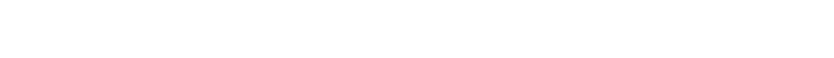

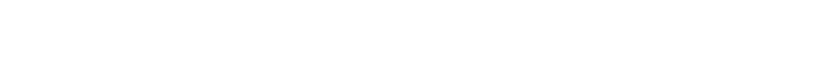

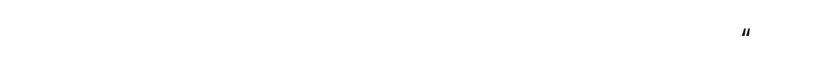

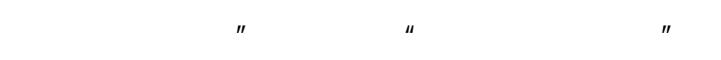

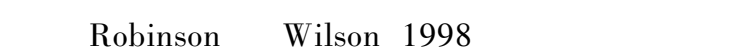

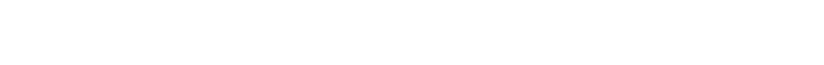

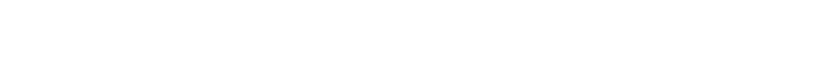

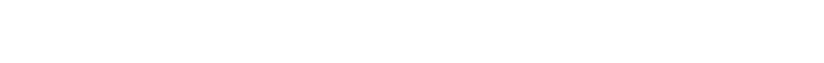

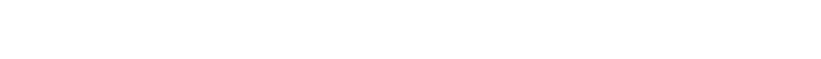

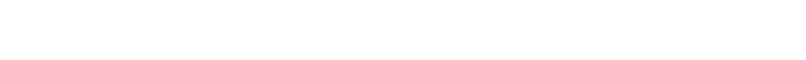

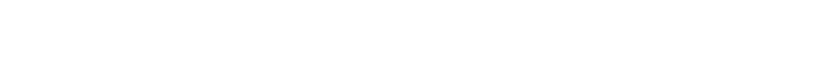

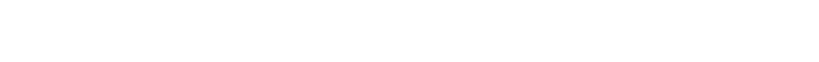

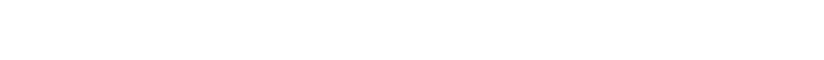

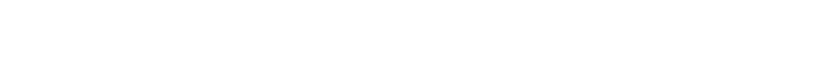

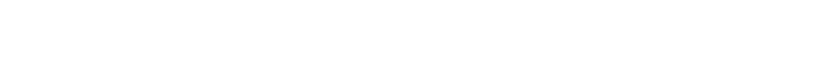

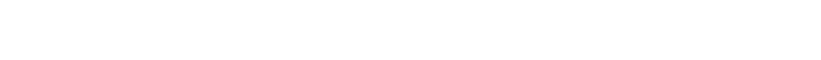

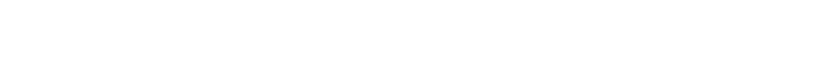

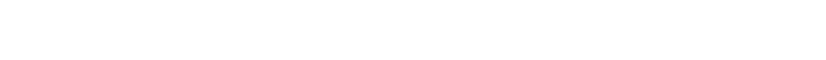

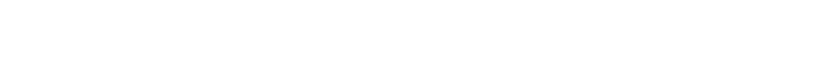

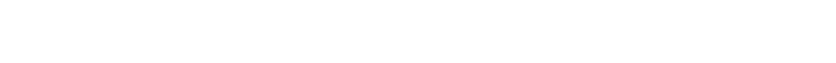

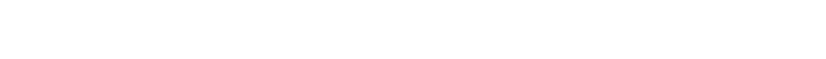

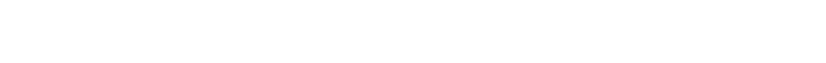

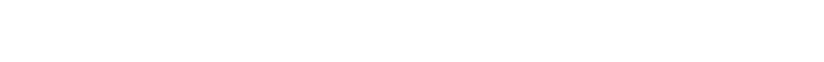

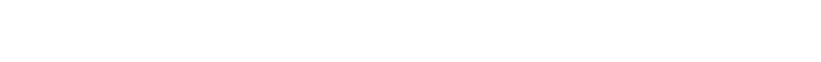

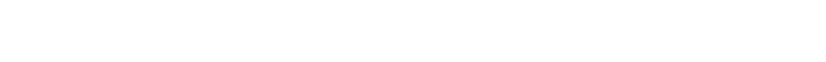

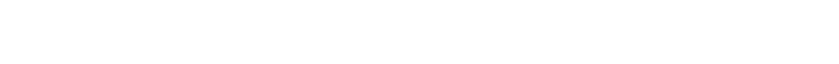

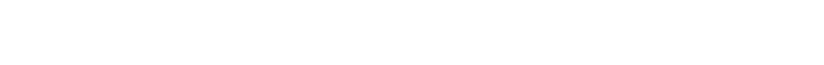

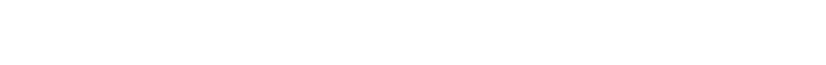

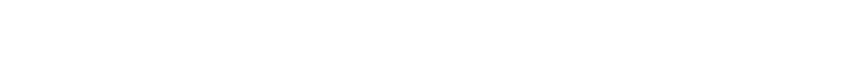

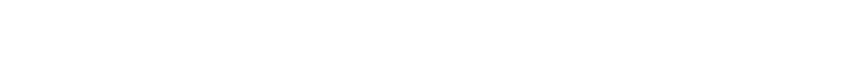

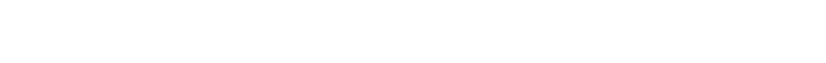

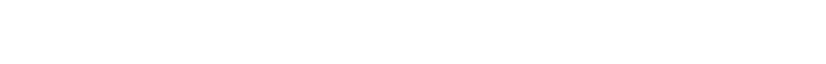

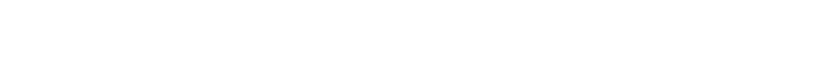

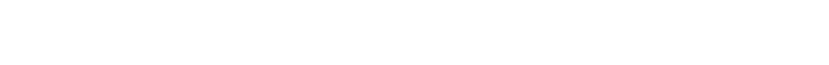

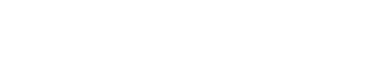

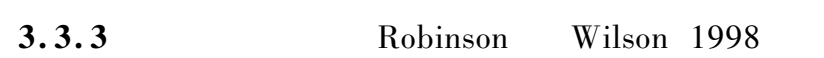

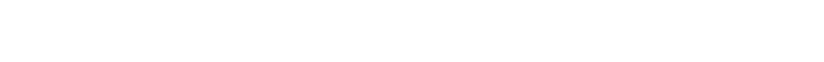

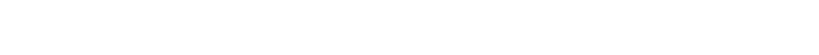

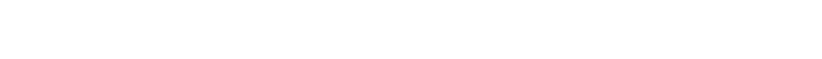

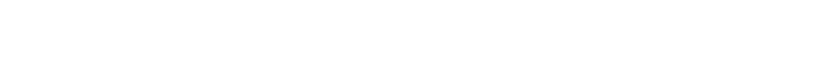

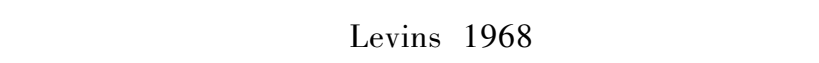

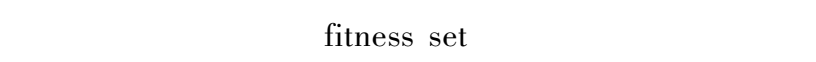

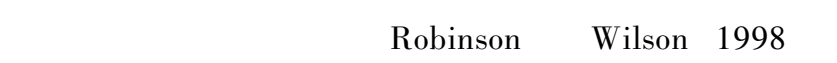

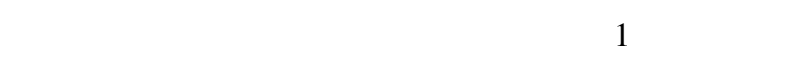

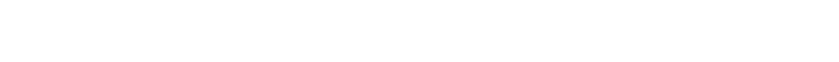

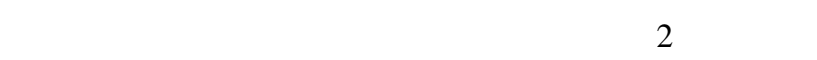

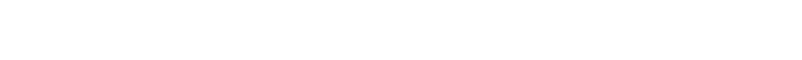

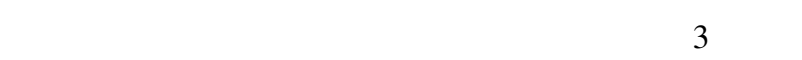

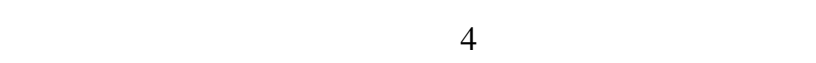

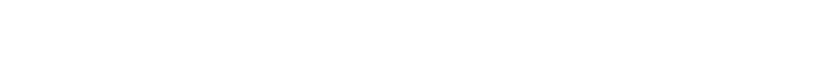

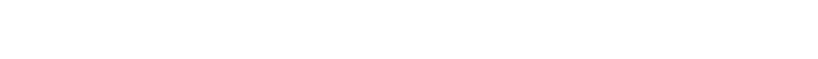

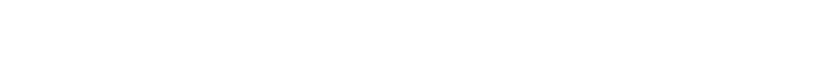

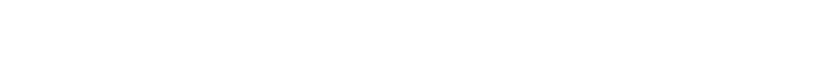

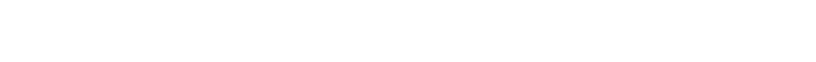

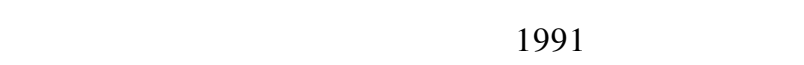

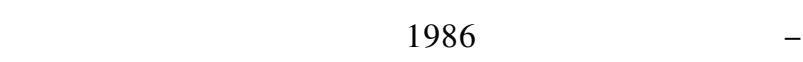

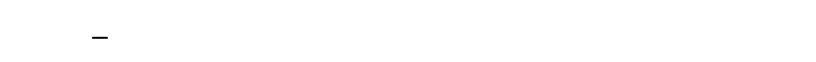
${ }^{1}$ ú\# ${ }^{1 / \alpha}$ 䄮

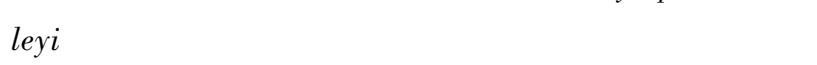

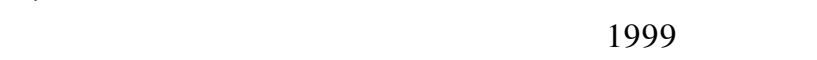

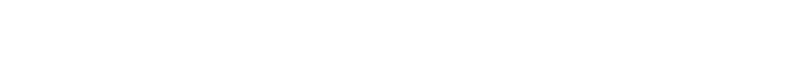

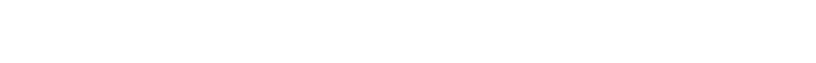

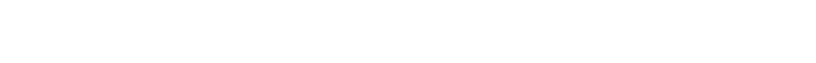

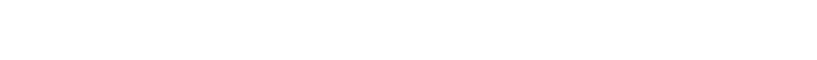

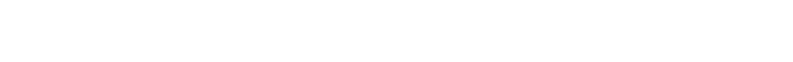

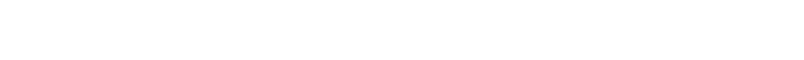

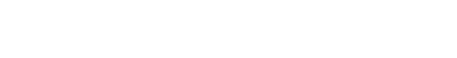

\section{1'向 ÊøÓ}

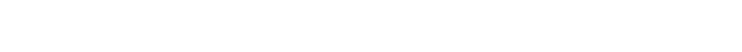

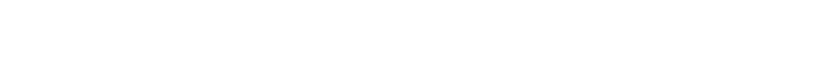

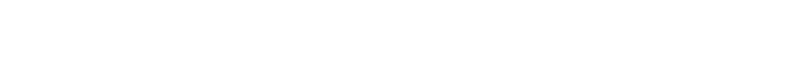

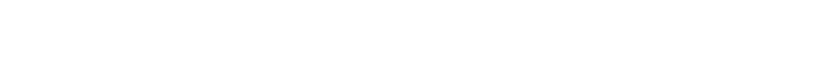

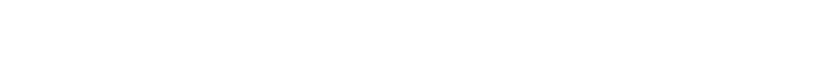

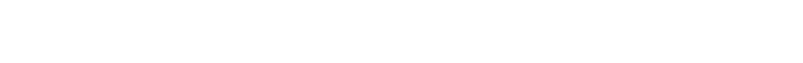

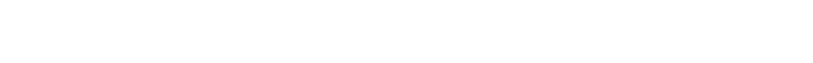

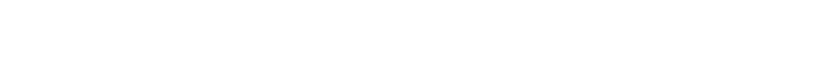

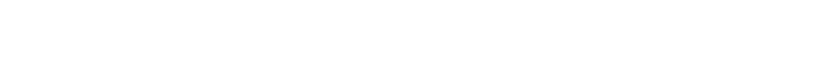

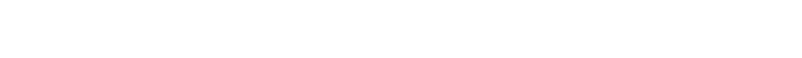




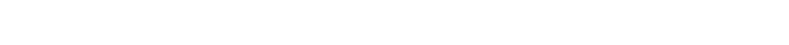

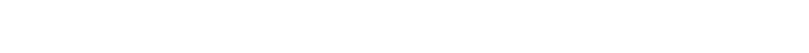

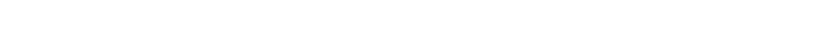

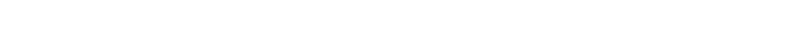

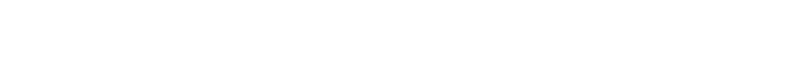

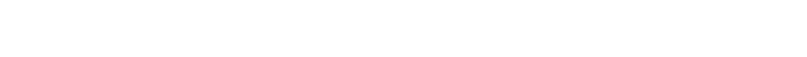

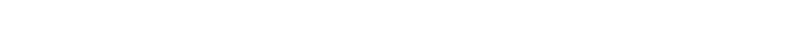

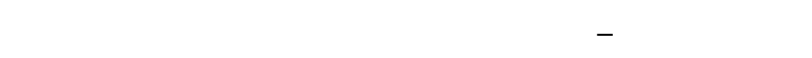

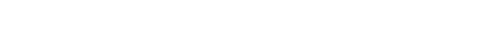

\section{${ }^{2} \hat{\imath} i^{1} \hat{\wedge} \hat{A} \ddot{A} x$}

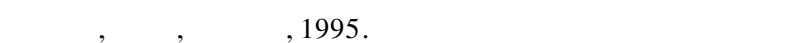

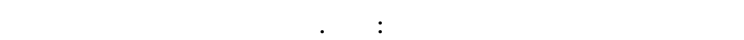
Éúl

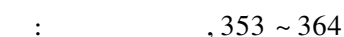

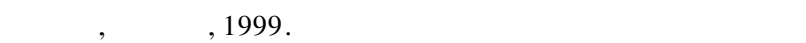

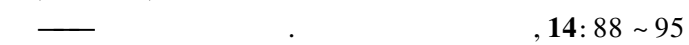

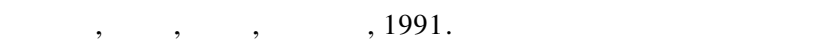

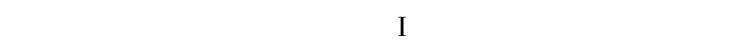

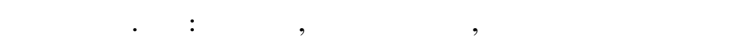

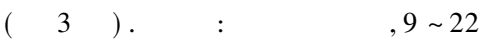

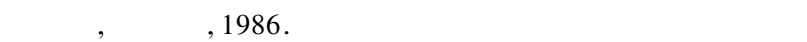

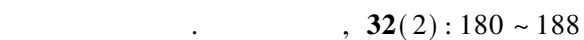

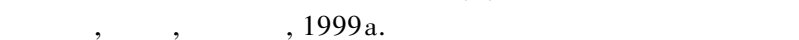

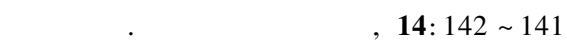

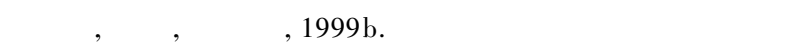

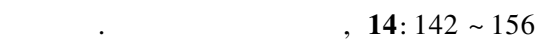

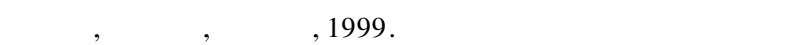

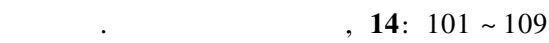

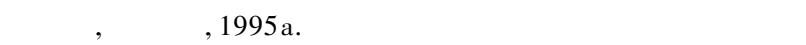

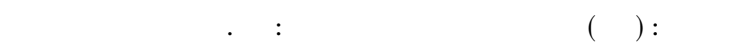

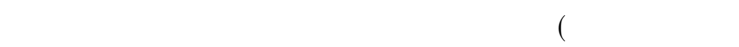

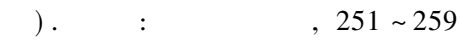

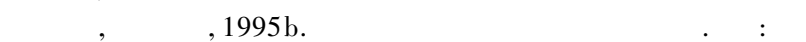

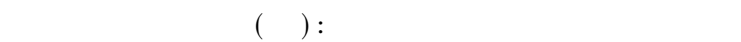

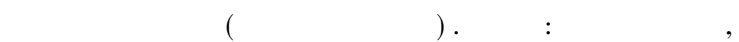
$251 \sim 259$

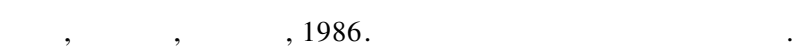

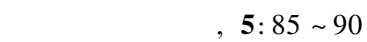

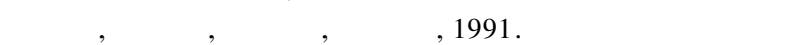

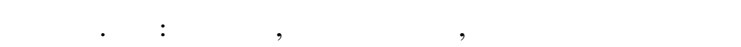

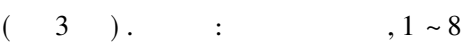

Aarssen L W, 1989. Competitive ability and species coexistence: a "plant's-eye" view. Oikos, 43: $369 \sim 375$

Aarssen L W, 1993. Ecolgical combining ability and competitive combining ability in plants: toward a general evolutionary theory of coexistence in systems of competition. American Naturalist, 122: 707 731

Abrams P A, 1988. Resource productivity ${ }^{a}$ consumer species diversity: simple models of competition in spatially heterogeneous environments. Ecology, 69: $1418 \sim 1433$
Abramsky Z, Rosenzweig M L, Pinshow B, Brown J S, Kotler B, and Mitchell W A, 1990. Habitat selection: an experimental field test with two gerbil species. Ecology, 71:2358 $\sim 2369$

Abramsky Z, Rosenzweig M L, Pinshow B, 1991. The shape of a gerbil isocline measured using principles of optimal habitat selection. Ecology, 72:329 340

Abramsky Z, Rosenzweig M L and Zubach A, 1992. The shape of a gerbil isocline: an experimental field study. Oikos, 63: $193 \sim 199$

Arthur W, 1982. The evolutionary consequences of interspecific competition. Advances in Ecological Research, 12: 127 187

Bengtsson J, Fagerstrom T, Rydin H, 1994. Competition and coexistence in plant communities. Trends in Ecology and Evolution, 9: $246 \sim 250$

Bertness M D, Hacker S D, 1994. Physical stresses and positive associations among marsh plants. American Naturalist, 144: $363 \sim 372$

Brown J S, 1989. Desert rodent community structure: a test of four mechanisms of coexistence. Ecological Monographs, 59: $1 \sim 20$

Chesson P L, 1986. Environmental variability and coexistence of species. In: Dimond J and Case T J (eds.), Community ecology. New York: Harper \& Row, $240 \sim 256$

Chitty D, 1960. Population processes in the vole and their relevance to general theory. Canadian Journal of Zoology, 38: $99 \sim 113$

de Jong G, 1982. The influence of dispersal pattern on the evolution of fecundity. Netherlands Journal of Zoology, 32:1 $\sim 30$

Dudgeon S R, Steneck R S, Davison I R and Vadas R L, 1999. Coexistence of similar species in a space-limited intertidal zone. Ecological Monographs, 69 (3):331 352

Epp G A and Aarssen L W, 1989. Predicting vegetation pattens from attributes of plant growth in grassland species. Canadian Journal of Botany, 67:2953 2959

Ewel J, 1983. Succession. In: Golley F B (ed.), Tropical rain forest ecosystems, A. Structure and function. Elsevier, Amsterdam, $217 \sim 223$

Fry G, Iles T D, 1972. The Cichlid Fishes of the Great Lakes of Africa. Oliver \& Boyd, Edinburgh

Grubb P J, 1977. The manitenance of species-richness in plant communities: the importance of the regeneration niche. Biology Review, 52: $107 \sim 145$

Grime J P, 1979. Plant Strategies and Vegetation Processes. Wiley, Chichester.

Hanski I, 1981. Coexistence of competitors in patchy environment with and without predation. Oikos, 37: $306 \sim 312$

Heard S B and Remer L C, 1997. Clutch-size behavior and coexistence in ephemeral-patch competition models. American naturalist, 150: $744 \sim 770$

Heinrich B and Raven P H, 1972. Energetics and pollination ecology. Science, 176: $597 \sim 602$

Holmes R T, Schults J C and Nothnagle P, 1979. Bird preda- 
tion on forest insects: an experiment. Science, 206: 462 463

Howard W E, 1960. Innate and environmental dispersal of individual vertebrates. American Mildly Naturalist, 63: 152 61

Kotler B P, Brown J S and Ubach A S, 1993. Mechanisms of species coexistence of optimal foragers: temporal partitioning by two species of sand dune gerbils. Oikos, 67:548 556

Levins R, 1968. Evolution in Changing Environments. New Jersey: Princeton University Press

MacArtur R H and Pianka E R, 1966. On optimal use of a Patchy environment. American naturalist, 100: $603 \sim 609$

MacArthur R H and Wilson E O, 1967. The Theory of Island Biogeography. Princeton, New Jersey: Princeton University Press

Malloch D W, Pirozynski K A and Ravan P H, 1980. Ecological and evolutionary significance of mycorrhizal symbioses in vascular plants (a review). Proceedings National Academy of Sciences USA, 77:2113 2118

May R M and MacArthur R H, 1972. Niche overlap as a function of environmental variability. Proceedings National Academy of Sciences USA, 69: $1109 \sim 1113$

Menge B A and Sutherland J P, 1976. Species diversity gradients: synthesis of the role of predation, competition, and temporal heterogeneity. American Naturalist, 110: 351 369

Miller T E, 1982. Community diversity and interactions between the size and frequency of disturbance. American Naturalist, 120: $533 \sim 536$

Munguira M L and Thomas J A, 1992. Use of road verges by butterfly and burnet populations, and the effects of roads on adult dispersal and mortality. Journal of Applied Ecology, 29: $316 \sim 329$

Murray B G, 1967. Dispersal in vetebrates. Ecology, 48:975 978

Nevo E, 1979. Adaptive convergence and divergence of subterranean mammals. Annual Review of Ecology and Systematics, 10: $269 \sim 308$

Paine R T and Levin S A, 1981. Intertidal landscapes: disturbance and the dynamics of pattern. Ecological Monographs, 51: $145 \sim 178$

Pearson D L, 1986. Community structure and species co-occurrence: a basis for developing broader generalizations. $\mathrm{O} i$ kos, $46(3): 419 \sim 423$

Remer L C and Heard S B, 1998. Local Movement and Edge Effects on Competition and Coexistence in EphemeralPatch Models. American naturalist, 152: 896 904

Robinson B W and Wilson D S, 1998. Optimal foraging, specialization, and a solution to Liem's Paradox. American naturalist, $151: 223 \sim 235$
Sale P F and Williams D McB, 1982. Community structure of coral fishes: are the patterns more then those expected by chance? American Naturalist, 120: $121 \sim 127$

Schoener T W, 1983. Field experiments on interspecific competition. American Naturalist, 122: $240 \sim 285$

Sevenster J G and van Aphen J J M, 1996. Aggregatoin and coexistence. II. A neotropical Drosophilae community. Journal of Animal Ecology, 65: $308 \sim 324$

Shmida A and Ellner S, 1984. Coexistence of plant species with similar niches. Vegetation, 58: $29 \sim 55$

Shorrocks B and Rosewell J, 1986. Guild size in drosophilids: a simulation model. Journal of Animal Ecology, 55: 527 541

Stephens D and Krebs J, 1986. Foraging Theory. Princeton, New Jersey: Princeton University Press

Taylor D R, Aarssen L W and Loehle C, 1990. On the relationship between $\mathrm{r} / \mathrm{K}$ selection and environmental carrying capacity: a new habitat temple for plant life history strategies. Oikos, 58: $239 \sim 250$

Tilman D, May R M, Lehman C L and Nowak M A, 1994. Habitat destruction and the extinction debt. London: $\mathrm{Na}$ ture, 371: $65 \sim 66$

Toda M J, Kimura M T and Tuno N, 1999. Coexistence mechanisms of mycophagous drosophilids on multispecies fungal hosts: aggregation and resource partitioning. Journal of Animal Ecology, 68: $794 \sim 803$

Weiher E, Clarke G D P and Keddy P A, 1998. Community assembly rules, morphological dispersion, and the coexistence of plant species. Oikos, 81(2): $309 \sim 323$

Whittaker R H, 1970. Communities and Ecosystems. London: Macmillan

Wiens L A, 1977. On competition and variable environments. American Science, 65: $590 \sim 597$

Wiens J A and Rotenberry J T, 1980. Patterns of morphology and ecology in grassland and shrubsteppe bird populations. Ecological Monographs, 50: $287 \sim 308$

Wilson S D and Tilman D, 1991. Components of plant competition along an experimental gradient of nitrogen availability. Ecology, 72: $1050 \sim 1065$

Witman J, 1987. Subtidal coexistence: storms, grazing, mutualism and the zonation of kelps and mussels. Ecological Monographs, 57: $167 \sim 187$

Wootton T, 1998. Effects of disturbance on species diversity: a multitrophic perspective. American Naturalist, 152: 803 825

Zovel M, 1992. Plant species coexistence $\mathbf{i}^{\mathrm{a}}$ the role of historical, evolutionary and ecological factors. Oikos, 65(2) : 314 $\sim 320$ 